\title{
INVESTIGATION OF RELATIONSHIP FOR INHOMOGENEOUS SPATIAL DISTRIBUTION OF THE UPPER ATMOSPHERE GLOW WITH THE GEOLOGICAL STRUCTURE OF THE CRUST
}

\author{
Yuriy K. Krakovetsky, Valentin Krakovetsky, Yuriy Y. Krakovetsky", Valeriy Parnachev, \\ Lev Popov
}

National Research Tomsk State University, 634050, Tomsk, Russia

\begin{abstract}
Authors propose a new method for studying of the physical properties of the Earth's crust, based on the effect of interaction of electromagnetic parameters of the magnetosphere, the ionosphere (the upper atmosphere), the atmosphere and the lithosphere. The research has shown that the spatial distribution of the night glow of the upper atmosphere in the zone of high and mid-latitudes is fragmented, sectional inhomogeneity is localized, stable, regardless of the magnitude of the geomagnetic disturbances, and are tied to ground coordinates.
\end{abstract}

\section{Introduction}

Nowadays one of the most promising areas of geological and geophysical studies is the remote sensing of the earth's crust structures. This is a very powerful tool to obtain such goals as the research of structural horizons of lithosphere and hydrosphere, localization of energetically active areas in the Earth's crust and hydrosphere, estimation of structure of tension-deformation conditions of various horizons of the Earth's crust. It can also help people with detection of destructions of the Earth's crust and earthquake prediction. There are even more opportunities: Earth crust based local objects zone-circular structure study, mineral deposit prognostication and search, exploration drilling design and others; differentiation degree increase for ecological pollution of atmosphere, water environment, and the Earth's surface; localization of pathogenic areas, land-survey tasks accomplishment. Due to importance of this method many scientists dedicated their work to research in this area, ex. Bedini E. [1], Khan S.D., Mahmood K. [2], Sabins F. [3]

According to the observations made in Norilsk, uneven distribution of auroras in the region was found. It is characterized by the presence of the local isolated minima and maxima, spatially and statistically coincident with the geological structures of the Earth's crust, including the structures of various geological structure - as the host an ore deposit (Norilsk and Talnakh ore nodes), and oil and gas fields $[4,5]$.

Glow inhomogeneities in the middle latitudes were experimentally registered in 1992 on the fields of the Middle Vasyugan. In the P-345 borehole area, the profile length

\footnotetext{
*Corresponding author: ukkrakov@yandex.ru
} 
of $150 \mathrm{~km}$ of $56^{\circ} \mathrm{N}$ was covered using a scanning photometer. The results of geophysical data on the profile imposed on the seismic data on the roof of the productive Priobye Bazhenov Formation of Middle and top of the Paleozoic basement showed satisfactory agreement.

\section{Equipment and methods}

According to the research on our study, the new method was developed for remote ionospheric-telluric profiling, based on a comparison of the spatial distribution of inhomogeneities in the glow of the ionosphere with the geological structure of the crust. The first step is to photograph the atmosphere at a wide-angled camera situated on the ground. After that, it is needed to form the contours luminescence intensity map with reference to the ground coordinates, which is going to be superimposed on the geological basis (Figure 1).

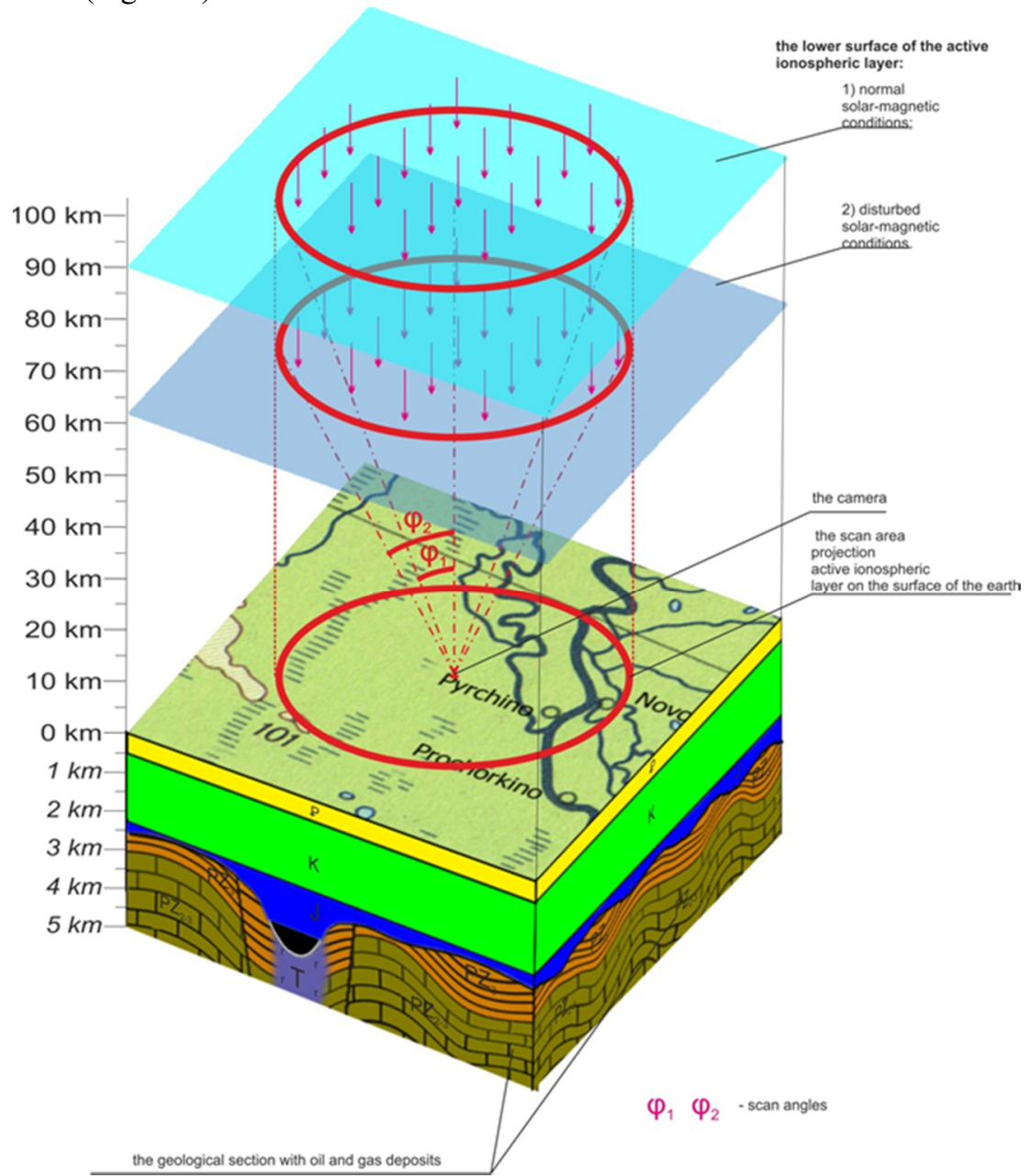

Figure 1. The scheme of works according to the method of ionospheric-telluric profiling.

Formerly photometry ionosphere emission was carried out on a black and white film and the processing was carried out manually by the operator, frame by frame. Now, with 
the advent of modern photographic and computer equipment processing capabilities greatly enhanced.

The inhomogeneities in the section of the upper atmosphere and the daytime sky in the middle latitudes can be detected via modern photomatrixes. However, the interpretation of this data is strongly hampered by powerful interferences. Overcoming of these problems requires the use of sophisticated mathematical libraries (such as spline interpolation) and serious computing power multithreading.

The use of modern matrix allows you to record the differences in the spectrum and the uneven brightness in increments of up to tens of meters (in terms of ground coordinates) and a resolution on the brightness of up to $0.01-0.001 \%$.

During the process of research we have been creating and constantly improving software that can automatically handle almost an unlimited number of photos, allowing you to quickly gain observation statistics for each shooting point.

The use of a camera with a wide-angle $\left(>90^{\circ}\right)$ for the day when the relevant atmospheric and meteorological conditions allows to capture and create the map of the daytime ionosphere glow irregularities in the scale of 1: $100000 \div 1: 200000$.

Many researchers in the work faces the need in photometry of different processes. For this purpose, special equipment is required, realizing various technologies of luminous flux conversion into the digital form for subsequent computer analysis. The most affordable is a CMOS-technology [6], implemented in a large number of professional photographic equipment from different manufacturers. When using such equipment users face a number of serious problems.

All the mass production photographic equipment is focused on getting quality pictures (feature films, documentaries), the most convenient for visual perception. Therefore, a large number of transformations is used for signals derived from the matrix of photosensitive elements to produce a target image in one of the standard RGB-format image. In the process of such a transformation a significant amount of useful information needed for scientific analysis of the latching process can be lost. Here are the negative factors:

\subsection{Debayer}

Matrix sensors are equally sensitive to all colors in the visible range, so to obtain a color picture they are covered with the mosaic color filter cover (most common Bayer filter, Figure 2) to form a $2 \times 2$ pixel cell is composed of red R, blue B and G1, and two green G2 cells. Each pixel receives only $1 / 4$ of color information. To get a "standard" tri-color RGBpixel it is necessary to restore the missing component based on the interpolation of color data from adjacent areas. This process is called debayer, it is based on complex algorithms that are the know-how of the photographic equipment manufacturer, and introduces serious distortions in the raw data read from each individual sensor [7]. In addition, the use of twice the number of "green" sensors (due to the peculiarities of human perception) should also be taken into account in the processing of metering data.

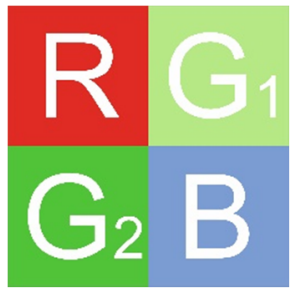

Figure 2. Bayer filter. 


\subsection{Rounding errors}

Standard RGB formats are the 24 or 48-bit ( 8 and 16 bits per channel, respectively). However, solar cells of matrixes from different vendors often have a different bit. For example, Canon EOS 5D Mark II that widely spread in the market - it's 14-bit [8]. Converting to the so-called "uncompressed» (TIFF or BMP) 24bit format will inevitably cause data loss, significant for the research.

The use of RAW-converters. All professional mass-produced photographic equipment allows to record in a file the unmodified data obtained directly from the image sensor. This is so-called RAW-format. Every producer has his own one. To work with it, you need to use the RAW-converters designed by the manufacturer to significantly expand the possibilities of photographers. Still, it does not allow to explore the source of sensor data "pixel by pixel".

Summarizing the mentioned problems, we can formulate the problem: getting the unchanged data per pixel (color sensor value and the value fixed by the signal) in the form of two-dimensional arrays of data for each separate color coordinates $\mathrm{x}$ and $\mathrm{y}$ (the starting point - the top left corner of the image). For example, we will use the RAW-file in the format * .CR2, obtained when using the camera Canon EOS 5D Mark II in the maximum available resolution of 21 megapixels.

\section{Software}

Since all the manufacturers of photographic equipment are hiding algorithms needed to work with their matrix, a large number of commercial and open source software for reading and RAW format data has emerged. Nowadays, most of them are based on the utility dcraw [9]. In our example we will use LibRaw [10] in development, distributed on a license-free basis [11]. Both products are oriented to the needs of professional photographers, but allows you to solve our problem (Figure 3).

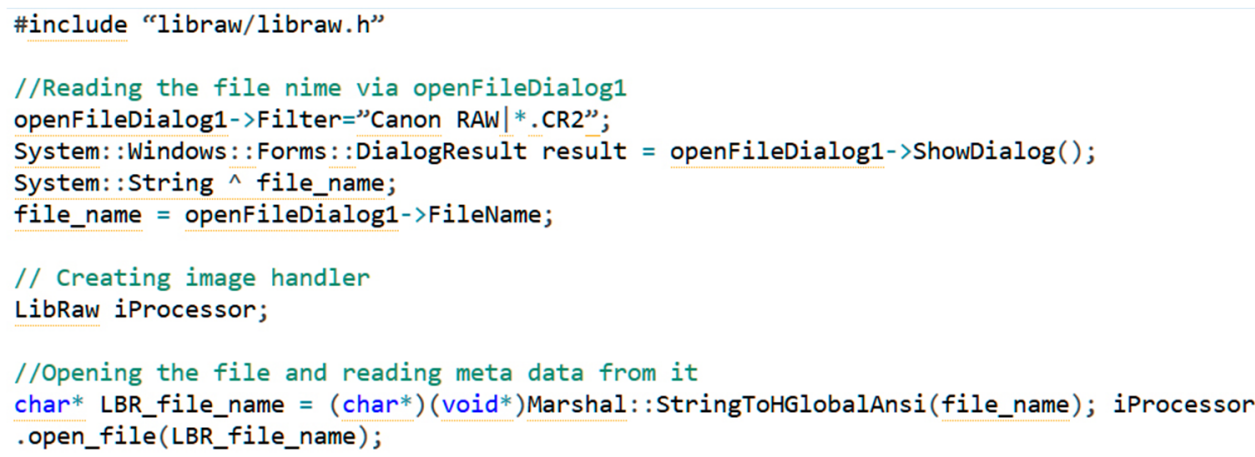

Figure 3. Connecting LibRaw library and opening a file. Learn more about its API $(\mathrm{C}++)$ on the project's official website [10].

\subsection{Peculiarities of RAW format}

Firstly, for our purposes it is necessary to take into account that 21 megapixels is the total number of sensors, which is made up of 5.25 million pixels of each color Bayer filter (RGGB). Secondly, the recording in RAW-file is carried out line by line. At the same time we do not know (according to the Canon documentation), how these elements are arranged 
in a conventional $2 \times 2$ box from left to right and from top to bottom: RGGB, RGBG or in a different order.

In other words, the odd lines of file will contain duplicate pairs RG1RG1 ... RG1, even BG2BG2 ... BG2 (there are 4 different versions). The final size of the file ( $\Rightarrow$ our twodimensional data sets) will be half the original size both vertically and horizontally (if the photo is on the "output", we will have a width 5616x3744, our arrays R, B, G1 and G2 will have a size of [2808, 1872] ) (Figure 4).

Third, when working with raw-file, you should keep in mind that it has a "black frame" containing a number of columns and rows with zero values of all pixels. For the definition of "usual size" and "the raw-image size" imgdata.sizes() method is responsible (parameters width and raw_width respectively) $[11,12]$.

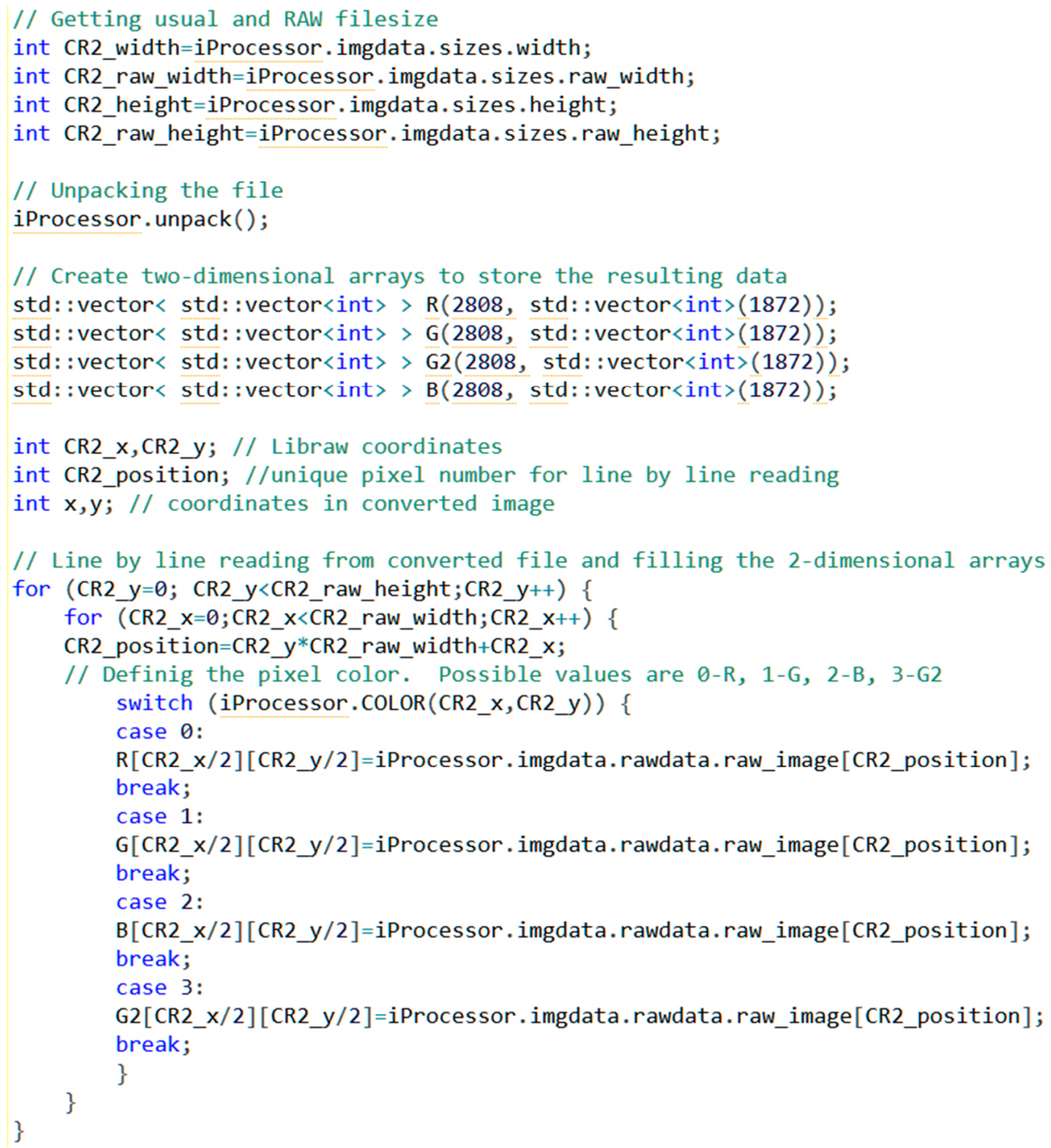

Figure 4. Filling the two-dimensional arrays of R, G1, G2, B. 
Currently, the authors are carrying out the experimental work on photometry atmosphere in middle latitudes (Tomsk Region). We obtained reliable data about irregularities of daytime atmosphere glow, reaching the first percents in different color channels (Figure 5).

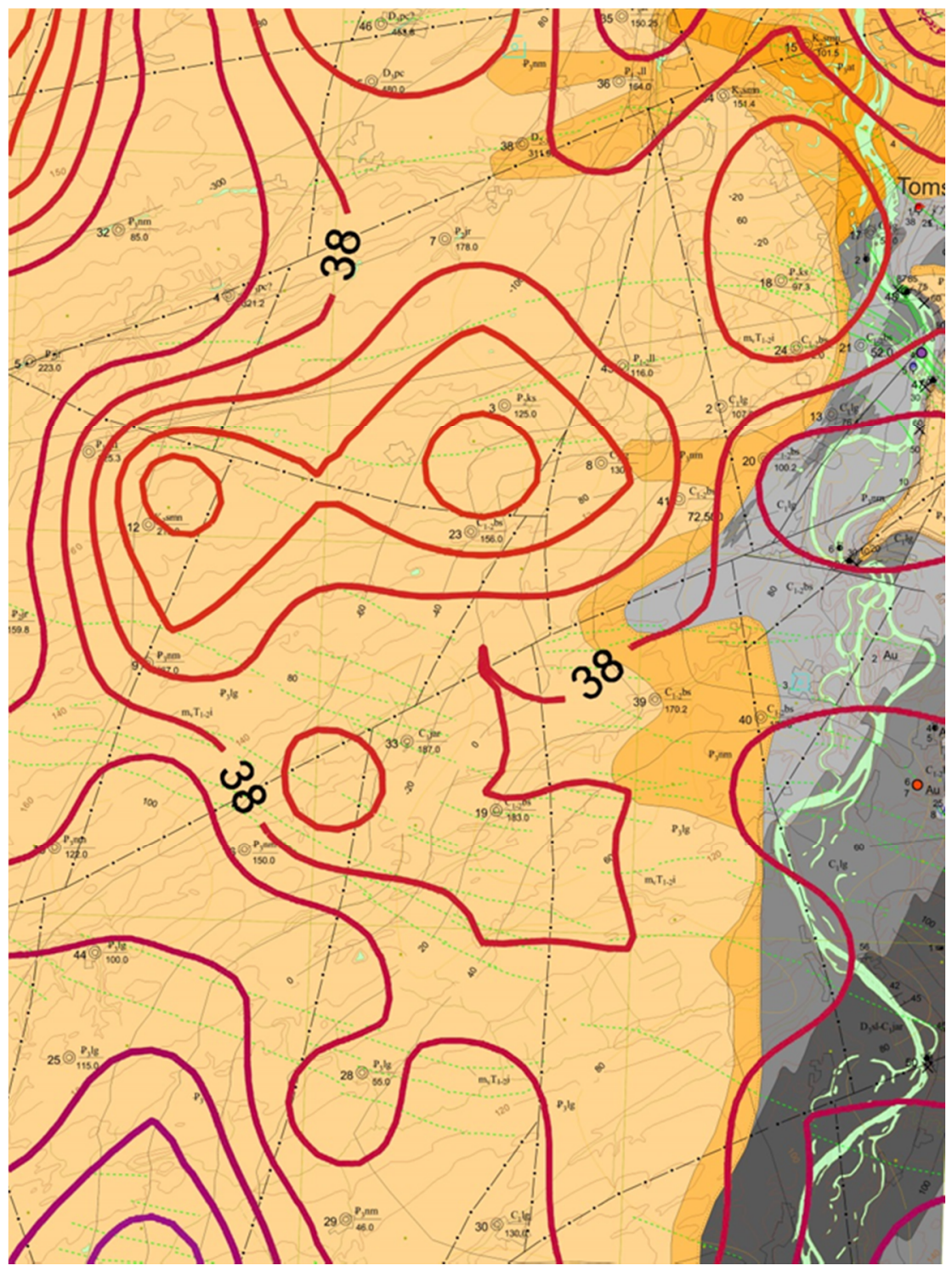

Figure 5. Map of the uneven distribution of the daytime sky illumination, the red (\%), Tomsk neighborhood, scale 1: 200000.

\section{Conclusions}

1. The new method for remote ionospheric-telluric profiling, based on a comparison of the spatial distribution of inhomogeneities in the glow of the ionosphere with the geological structure of the crust, was invented.

2. A program for photometric data processing was offered. 
3. The result is two-dimensional arrays of four color components under a Bayer filter data, are not subjected to any treatment. This information can be used in scientific research, demanding precision measurement at metering.

\section{References}

[1] E. M. Bedini, Remote Sensing of Environment 113, 1208 (2009) doi: 10.1016/j.rse.2009.02.007

[2] S.D. Khan, K. Mahmood, Earth-Science Reviews 89, 135 (2008) doi: $0.1016 /$ j.earscirev.2008.04.004

[3] F.F. Sabins, Remote Sensing - Principles and Interpretation (Waveland Press, Long Grove, IL, 2007)

[4] Y.K. Krakovetzkiy, M.V. Gouhberg, V.A. Pilipenko, L.N. Popov, International Union of Geodesy and Geophysics (IUGG), Abstracts V 1. - P 389 (1987)

[5] L.N. Popov, Yu.K. Krakovetskiy, M.B. Gokhberg, V.A. Pilipenko, Physics of the Earth and Planetary Interiors 57 , $115 \quad$ (1989) doi: 10.1016/0031-9201(89)90221-5

[6] CMOS (Complementary Metal-Oxide Semiconductor) URL: http://www.engineersgarage.com/articles/what-is-cmos-technology

[7] RGB "Bayer" Color and MicroLenses - Silicon Imaging URL: http://www.siliconimaging.com/RGB\%20Bayer.htm

[8] Canon corp., Canon EOS 5D Mark II (2015) URL: http://www.canon.ru/

[9] Dcraw project, the official site of dcraw project. (2016) URL: http://www.cybercom.net/ dcoffin/dcraw/index.html

[10] LibRaw project, The official site (2016) URL: http://www.libraw.su/

[11] LibRaw project, LibRaw documentation (2016) URL: http://www.libraw.su/docs.

[12] LibRaw project, C++ API for LibRaw project (2016) URL: http://www.libraw.su/docs/API-CXX-rus.html 